Return to the Manage Active Submissions page at http://spie.org/submissions/tasks.aspx and approve or disapprove this submission. Your manuscript will not be published without this approval. Please contact author_help@spie.org with any questions or concerns.

\title{
NASA's Optical Communications Program for 2015 and Beyond
}

\author{
Donald M. Cornwell*a \\ Technology Division, Space Communications and Navigation (SCaN) Program, NASA \\ Headquarters, 300 E Street Southwest, Washington, DC USA 20546
}

\begin{abstract}
NASA's Space Communications and Navigation (SCaN) Program at NASA Headquarters is pursuing a vibrant and wide-ranging optical communications program for future planetary and near-Earth missions following the spectacular success of NASA's Lunar Laser Communication Demonstration (LLCD) from the Lunar Atmosphere and Dust Environment Explorer (LADEE) spacecraft orbiting the Moon in 2013. This invited paper will discuss NASA's new laser communications missions, key scenarios and details, and the plans to infuse this new technology into NASA's existing communications networks.
\end{abstract}

Keywords: Free-space laser communications, optical communications, high data-rate, deep space, near-Earth

\section{NASA'S HISTORIC LLCD MISSION AND ITS IMPACT}

Today's radio-frequency (RF) communications are the bottleneck for returning more science data from NASA missions, especially those from beyond geostationary Earth orbit (GEO). Free-space optical communication (FSOC) offers the promise of breaking through this bottleneck by using laser transmitters at frequencies that are 10,000 times higher than those of RF and thus allowing many more bits of information to be imparted to the beam. Similarly, the commensurately shorter wavelength of an optical system allows for a narrower transmitted beam divergence that translates to more concentrated communications power at the receiver and lower transmit power to and from smaller, lighter apertures.

NASA's historic LLCD mission (depicted in Figure 1) demonstrated the potential of this new technology with data downlink rates of up $622 \mathrm{Mbps}$ from 400,000 $\mathrm{km}$ in lunar orbit [1]. This is almost an order of magnitude higher data rate from the Moon than our best Ka-band radio. LLCD also demonstrated that optical could deliver a more efficient communication system, since LLCD used only half the mass $(30.7 \mathrm{~kg})$ and $25 \%$ less power $(90 \mathrm{~W})$ than LRO $(61 \mathrm{~kg}$ and $120 \mathrm{~W}$, respectively). Such improvements in the returned data volume and the size, weight and power (SWaP) are extremely attractive to NASA's future planetary missions as they plan to greatly increase the total science return by both expanding the volume of data collected and devoting more resources to the onboard instruments.

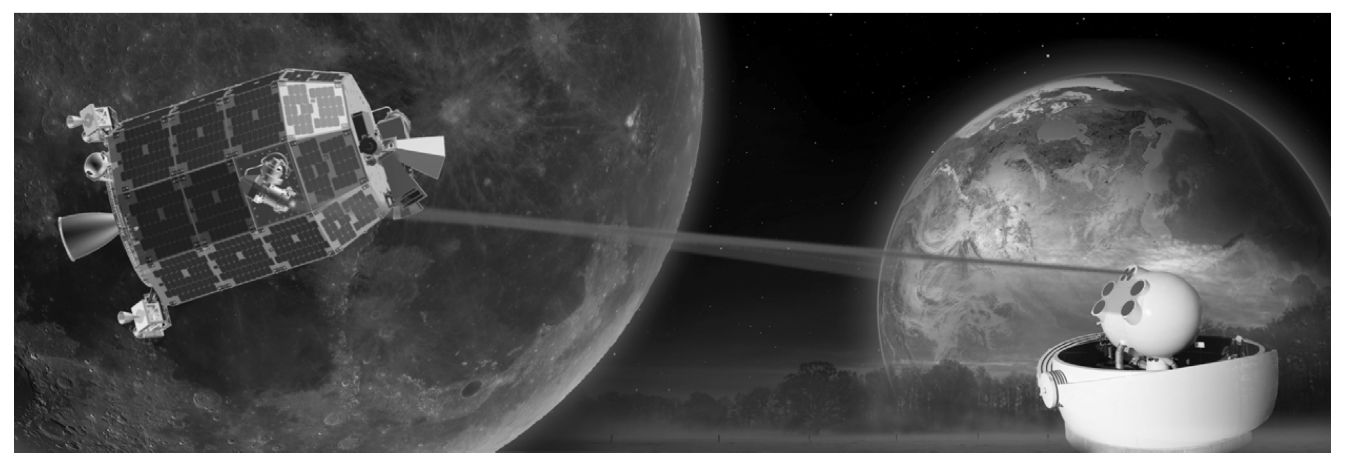

Figure 1. NASA's first historic, dedicated laser communications demonstration: the LLCD Mission on LADEE in 2013.

LLCD also demonstrated a 20 Mbps uplink (which was used to loop back error-free high-definition video to and from the Moon, an extremely attractive capability for future human exploration missions) and also provided simultaneous centimeter-class precision ranging to the spacecraft, which can be used to improve the gravity models of planetary bodies.

The real breakthrough of the LLCD demonstration, however, was the spectacular success from the standpoint of its essentially-flawless operations that allowed the system to be used to return real, high-value science data from LADEE's 


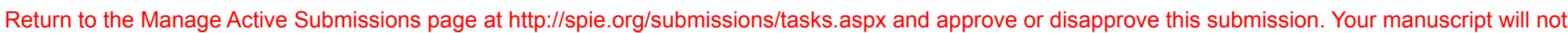
be published without this approval. Please contact author_help@spie.org with any questions or concerns.

instruments that were investigating the environment of the Moon. The LLCD space terminal and primary ground terminal (both designed, built and operated by the Massachusetts Institute of Technology's Lincoln Laboratory) demonstrated near-instantaneous acquisition followed by closed-loop tracking of the 15 microradian uplink and downlink beams on every pass while delivering error-free data under a range of conditions, including through thin clouds (thus demonstrating significant system margin), during daylight while the LADEE spacecraft was within 3 degrees of the Sun, and also while LADEE and the Moon were less than 5 degrees above the horizon [2]. The LLCD space terminal also demonstrated regular link "handovers" in less than 2 minutes between the primary and two alternate ground terminals, which were provided by NASA's Jet Propulsion Lab (JPL) at Table Mountain, CA and by the European Space Agency (ESA) in Tenerife, Spain. LLCD operations continued from mid-October 2013 to midNovember 2013, after which the space terminal was placed in "cold storage". LLCD was revived without issue for extended operations in March 2014 and ended with the impact of the LADEE spacecraft on the Moon in April 2014.

NASA management in both the Human Exploration and Operations Mission Directorate (HEOMD) and Science Mission Directorate (SMD) understood that LLCD had demonstrated operational performance and capabilities that were comparable to those of NASA's venerable, RF-based Deep Space Network (DSN). LLCD had made the case for laser communications (lasercomm) as a viable operational complement to RF rather than always being the "technology of tomorrow" for NASA's networks.

\section{NASA'S NEXT STEPS: LASER COMMUNICATIONS FOR DEEP SPACE AND PLANETARY EXPLORATION}

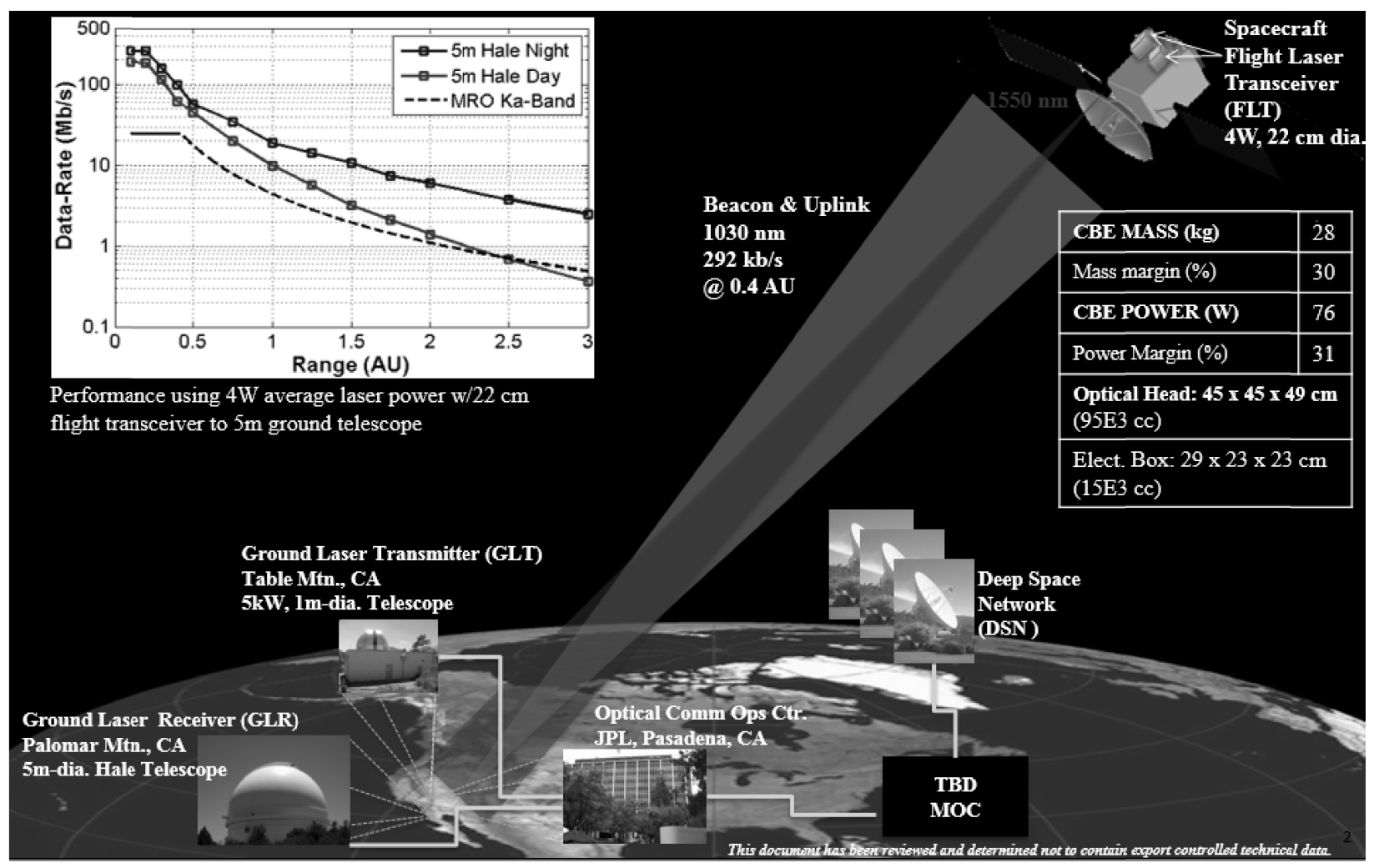

Figure 2. NASA JPL's Deep Space Optical Communications (DSOC) Project, providing up to 250 Mbps from Mars at opposition (Figure courtesy of Dr. Abi Biswas, NASA JPL). 
Return to the Manage Active Submissions page at http://spie.org/submissions/tasks.aspx and approve or disapprove this submission. Your manuscript will not be published without this approval. Please contact author_help@spie.org with any questions or concerns.

LLCD's historical success has given new impetus to NASA's prior Deep-space Optical Terminal (DOT) effort at NASA JPL [3]. Specifically, NASA's Space Technology Mission Directorate (STMD) and SMD have now teamed up with the HEOMD SCaN Program to take the re-named Deep Space Optical Communications (DSOC) terminal to Technology Readiness Level (TRL) 6 by the end of FY17 so that it can be flown as government-furnished equipment (GFE) on the upcoming Discovery 2020 mission.

As shown in Figure 2, DSOC is designed to work from near-Earth asteroids on out to Jupiter and could deliver data at over $250 \mathrm{Mbps}$ from Mars at opposition $(0.42 \mathrm{AU}$, or $63 \mathrm{Mkm})$ as illustrated in Figure 2, all while consuming $28 \mathrm{~kg}$ of mass and using $76 \mathrm{~W}$ of power. DSOC, however, will have additional challenges compared to LLCD, including a link that is 1000x further away (with $60 \mathrm{~dB}$ more link loss), a kilowatt-class uplink beam from the ground, a photon-counting detector array on the spacecraft to see that uplink beam, and a requirement for an order-of-magnitude improvement for inertially-stabilized beam pointing and larger point-ahead angles for the downlink beam. DSOC will also require larger receiver apertures on the ground than LLCD - development of a 12 meter telescope is currently under study [4] although current plans are to use the 5 meter Hale telescope on Mount Palomar to deliver more than 100 Mbps from Mars at opposition. Note that the highest RF data rate demonstrated from Mars is $6 \mathrm{Mbps}$ from the Ka-band transmitter on the Mars Reconnaissance Orbiter (MRO), so DSOC represents an order-of-magnitude improvement over what the Mars science community has received to date.

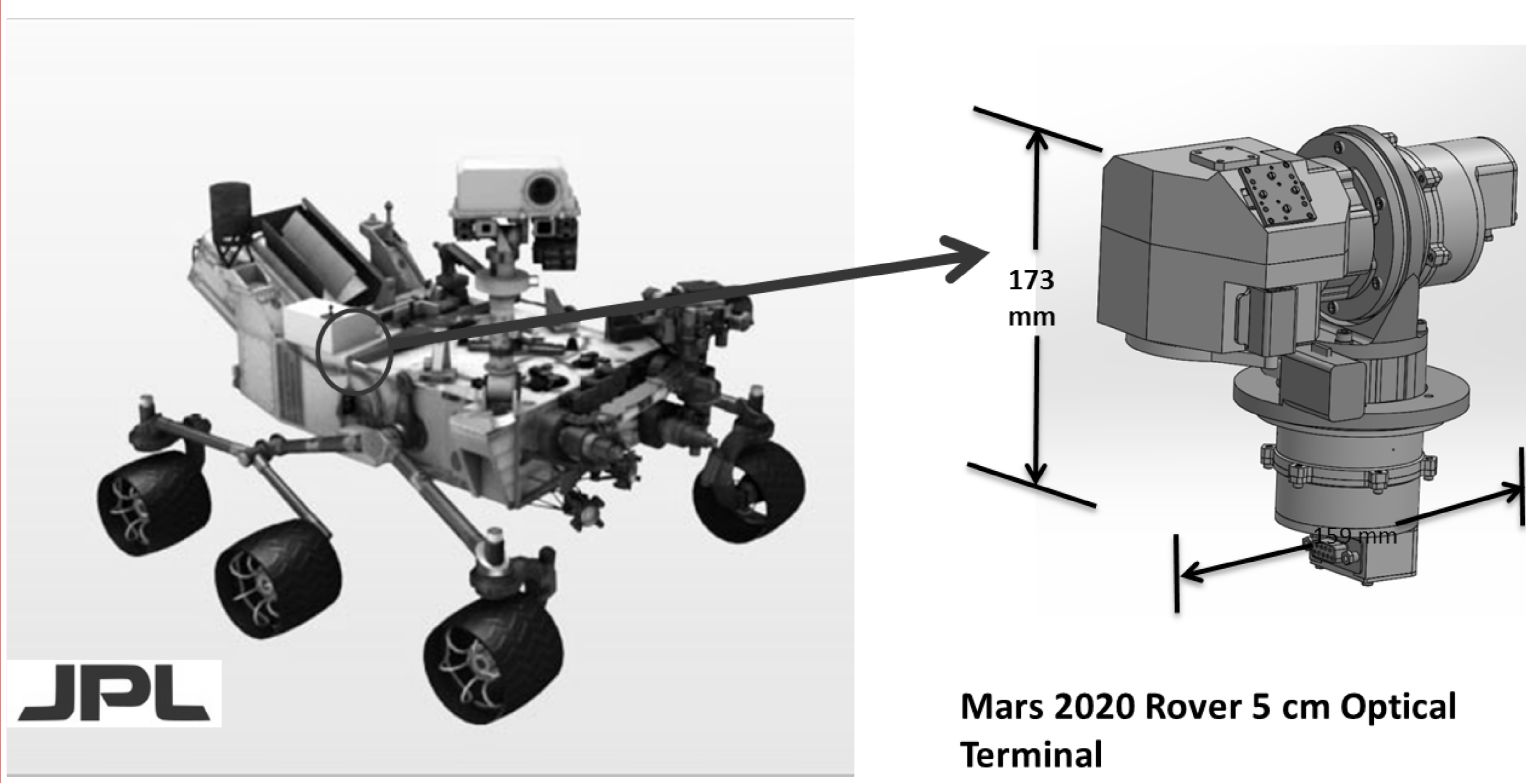

Figure 3. Proposed optical communications terminal on the Mars 2020 rover for both relay and DTE laser links (figure courtesy of Dr. Joseph Kovalik, NASA JPL).

The SCaN Program has also supported a recent NASA JPL study to place a very small $(\sim 6 \mathrm{~kg}, 50 \mathrm{~W})$ lasercomm terminal on the Mars 2020 rover as shown in Figure 3. This terminal could communicate to an optical relay terminal on a Mars orbiter at up to $20 \mathrm{Mbps}$ (which of course requires such a terminal) or, interestingly, it could provide a "direct-toEarth" (DTE) link from the Martian surface at up to $200 \mathrm{kbps}$, which is an order of magnitude better than the current Xband RF DTE link. This system will be based on a small $(5 \mathrm{~cm})$ transmit aperture and may find additional applications in low-Earth-orbit (LEO)-to ground applications for near-Earth lasercomm as well. 
Return to the Manage Active Submissions page at http://spie.org/submissions/tasks.aspx and approve or disapprove this submission. Your manuscript will not be published without this approval. Please contact author_help@spie.org with any questions or concerns.

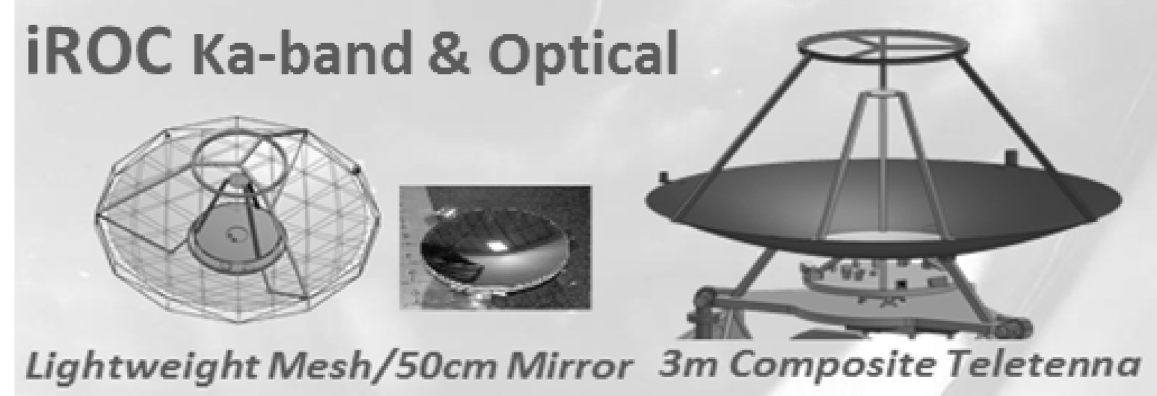

Figure 4. The integrated Radio and Optical Communications (iROC) terminal, a technology investigation to merge Ka-band $\mathrm{RF}$ and $1550 \mathrm{~nm}$ optical communications within the same size, weight, and power (SWaP) of today's current RF systems. (Figure courtesy of Dr. Daniel Raible, NASA GRC).

Finally, NASA's Glenn Research Center (GRC) is investigating a hybrid RF-optical system [5], known as the integrated Radio and Optical Communication (iROC, depicted in Figure 4), which will incorporate a body-pointed 3 meter RF mesh antenna with a $30 \mathrm{~cm}$ optical telescope, while sharing an integrated software-defined modem for both Ka-band and laser communications. This research effort is currently low TRL (2 to 3) but poses intriguing possibilities for planetary explorers if the SWaP of the hybrid system can stay within the SWaP of today's RF deep space terminals.

\section{NASA'S NEXT STEPS: LASER COMMUNICATIONS FOR THE NEAR-EARTH ENVIRONMENT}

NASA's debut mission for a low-Earth-orbit (LEO) DTE laser communications experiment is the Optical PAyload for Lasercomm Science (OPALS), which was installed on the International Space Station (ISS) in 2014 [6]. OPALS was developed and delivered as part of an early career training program for new employees at NASA's JPL, which meant that even the mission project management was required to be within three years of starting their professional careers. OPALS was able to complete many optical-based data downlinks at data rates up to $50 \mathrm{Mbps}$, based on a payload using primarily commercial off-the-shelf (COTS) components, and will soon be used as an orbiting test source for newlyinstalled adaptive optics on the NASA JPL $1 \mathrm{~m}$ telescope on Table Mountain in California (which had also successfully served as one of the LLCD ground stations). NASA is considering other LEO DTE missions to demonstrate laser communications downlinks that exceed 10 gigabits per second.

NASA is also developing laser communications technology for relaying terabits of data per day between points on the ground through satellites in the near-Earth environment. NASA's next major technology mission is the Laser Communications Relay Demonstration (LCRD) [7], which is being built by NASA's Goddard Space Flight Center with a space terminal that uses two of the three component modules derived from MIT Lincoln Laboratory's design for LLCD. The gimbaled $10.7 \mathrm{~cm}$ diameter optical telescope and pointing, acquisition, and tracking (PAT) controller are specifically derived from the space-qualified LLCD design. However, LCRD will use a new, multi-rate modem (MRM) to generate a $1.244 \mathrm{Gbps}$ (user rate) differential phase shift-keying (DPSK) modulated beam at $1550 \mathrm{~nm}$. The choice of this wavelength allows for commercially available erbium-doped fiber amplifier (EDFA) technology to be used to amplify the signals, both for high-power transmission $(0.5 \mathrm{~W}$ average power) and low-noise pre-amplification in the receiver. LCRD will fly two space terminals connected via a high-speed electronic switch that will provide data processing and also serve as an interface to the host spacecraft, which is a commercial telecommunications satellite in geostationary orbit (GEO) currently being built by Space Systems Loral of Mountain View, CA.

The LLCD ground stations at White Sands, New Mexico and on Table Mountain in California will both be upgraded with adaptive optics for use on LCRD. This upgrade will allow for coupling of the downlinked LCRD signals from GEO into single-mode optical fibers, which is required since demodulation of the DPSK signal requires spatial coherence at the receiver. Full user-rate $(1.244 \mathrm{Gbps})$ duplex communications will be supported between each ground station and the space terminals on the LCRD payload in GEO. A $1 / 2$ rate error correcting code (on the uncoded DPSK modulation rate of $2.88 \mathrm{Gbps}$ ) and a channel interleaver are used to deal with signal dropouts due to deep atmospheric fades. 


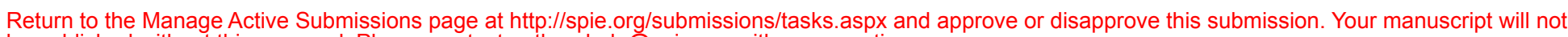
be published without this approval. Please contact author_help@spie.org with any questions or concerns.

Finally, LCRD is funded as a two-year demonstration (versus the two month demonstration for LLCD) with the fundamental goal of building long-term, multi-year operational experience and understand of laser communications systems. This includes long-term measurements and monitoring of atmospheric and meteorological conditions at each site and using that data, for example, to plan for fast handovers between the two ground stations (where one may be cloudy while the other is clear). Such experience will be key for further demonstrating the viability of this technology to serve NASA's communications needs in the near future.

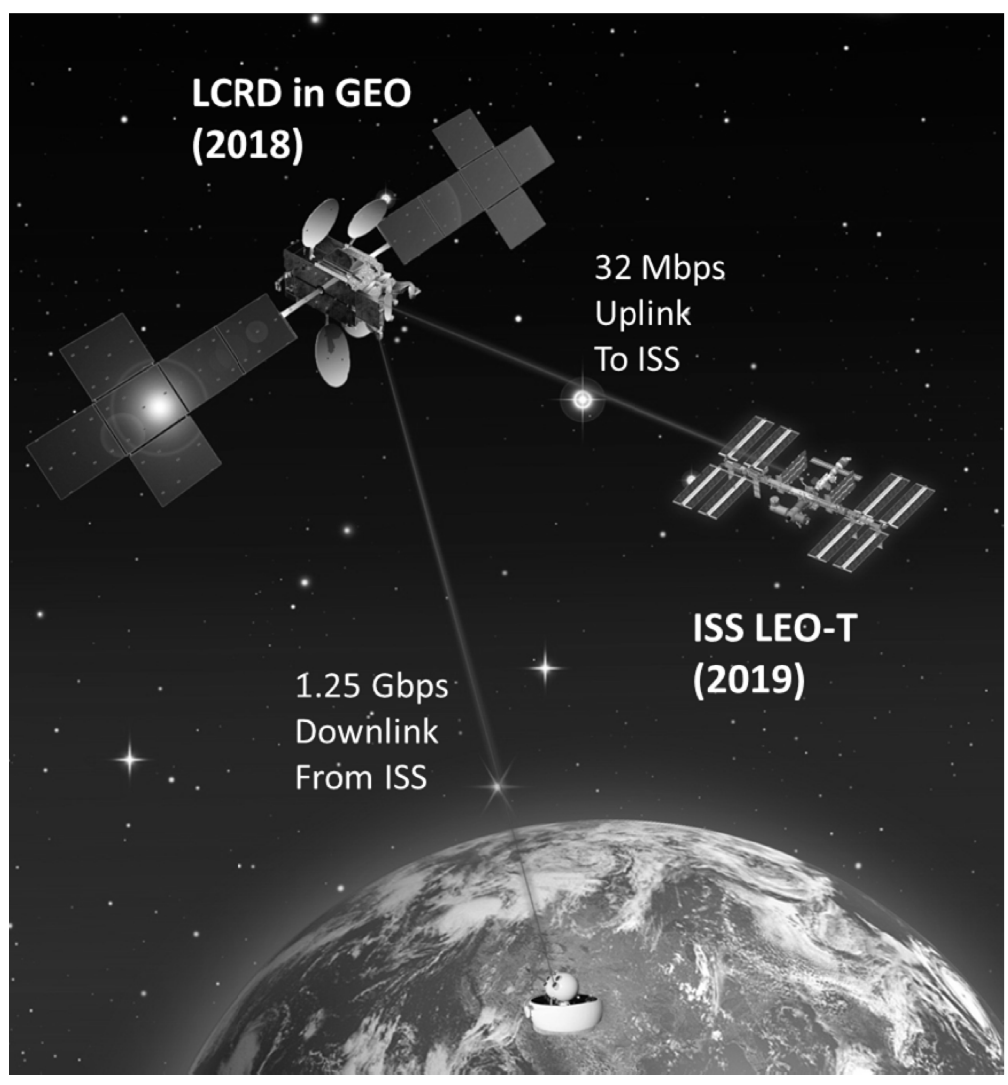

Figure 5. The LCRD and ISS LEO-T Missions for demonstrating near-Earth high-bandwidth optical relays.

Currently, LCRD is planned to relay data between two ground terminals. However, NASA has tentative plans to place the LCRD-derived Low-Earth Orbit Terminal (LEO-T) on the International Space Station (ISS) to fully demonstrate relay from LEO through the LCRD terminals in GEO and then to the ground (as depicted in Figure 5) as an optical analog to our current RF-based Tracking and Data Relay System (TDRS). NASA eventually plans to offer these services to our next generation of Earth-orbiting scientific satellites to download ever more data from new, high-resolution instruments.

Finally, NASA is committed to building a network of optical ground stations to support these missions. We are currently performing studies to build additional stations in addition to the two NASA stations ( $1 \mathrm{~m}$ apertures) used for LLCD. We are currently evaluating many sites around the world to identify the clearest skies possible to support optical communications on NASA's deep space and near-Earth missions of the future.

\section{REFERENCES}

[1] Boroson, D. M., Robinson, B. S., Murphy, D. V., Burianek, D. A., Khatri, F., Kovalik, J. M., Sodnik, Z., and Cornwell, D. M., "Overview and results of the Lunar laser Communication Demonstration," SPIE LASE, 89710S89710S, International Society for Optics and Photonics (2014). 
Return to the Manage Active Submissions page at http://spie.org/submissions/tasks.aspx and approve or disapprove this submission. Your manuscript will not be published without this approval. Please contact author_help@spie.org with any questions or concerns.

[2] Murphy, D. V., Kansky, J. E., Grein, M. E., Schulein, R. T., Willis, M. M., and Lafon, R. E., "LLCD operations using the Lunar Lasercom Ground Terminal," SPIE LASE, 89710V-89710V, International Society for Optics and Photonics (2014).

[3] Hemmati, H., Farr, W. H., Biswas, A., Birnbaum, K. M., Roberts, W. T., Quirk, K., and Townes, S., "Deep-space optical terminals (DOT)," SPIE LASE, 79230C-79230C, International Society for Optics and Photonics (2011).

[4] Hamid Hemmati, Abhijit Biswas and Ivan Djordevic, "Deep-Space Optical Communications: Future Perspectives and Applications," Proceedings of the IEEE, 99(11), 2020-2039, (2011).

[5] Raible, D., Robert R. Romanofsky, James M. Budinger, Jennifer M. Nappier, Alan G. Hylton, Aaron J. Swank, and Anthony L. Nerone. "On the Physical Realizability of Hybrid RF and Optical Communications Platforms for Deep Space Applications." In AIAA International Communications Satellite Systems Conference (2014).

[6] Oaida, Bogdan V., William Wu, Baris I. Erkmen, Abhijit Biswas, Kenneth S. Andrews, Michael Kokorowski, and Marcus Wilkerson. "Optical link design and validation testing of the Optical Payload for Lasercomm Science (OPALS) system." SPIE LASE, pp. 89710U-89710U. International Society for Optics and Photonics (2014).

[7] Edwards, B. L., Israel, D., Wilson, K., Moores, J., and Fletcher, A., "Overview of the laser communications relay demonstration project," Proceedings of SpaceOps, 11-15 (2012). 